\begin{tabular}{|c|l|}
\hline Title & A nnealing method for operating quantum-cellular-automaton sy stems \\
\hline Author(s) & A kazawa, M.; A memiya, Y.; Shibata, N. \\
\hline Citation & $\begin{array}{l}\text { Journal of A pplied Physics, 82(10), 5176-5184 } \\
\text { https://doi.org/10.1063/1.366322 }\end{array}$ \\
\hline Issue Date & 1997-11-15 \\
\hline Doc URL & http://hdl.handle.net/2115/5746 \\
\hline Rights & Copyright $\odot$ 1997 A merican Physical Society \\
\hline Type & article \\
\hline File Information & JAP82-10.pdf \\
\hline
\end{tabular}

Instructions for use 


\title{
Annealing method for operating quantum-cellular-automaton systems
}

\author{
M. Akazawa ${ }^{\text {a) }}$ and Y. Amemiya \\ Faculty of Engineering, Hokkaido University, Kita 13, Nishi 8, Sapporo 060, Japan \\ N. Shibata \\ Dai-Nippon Printing Co., Ltd., Nisbi-Gotanda 3-6-21, Shinagawa-ku, Tokyo 141, Japan
}

(Received 25 November 1996; accepted for publication 1 August 1997)

\begin{abstract}
We propose an annealing method as an effective way of operating quantum-cellular-automaton (QCA) systems, which are devices for computation that utilize the minimum energy state of electrons in a quantum cell system. A QCA system has an energy function with many local minima and therefore cannot be operated as desired if placed under the conditions of a thermodynamically open system. Accordingly, for successful operation of a QCA system (i.e., making the QCA system converge successfully to its minimum-energy state), we propose a method of operation based on the concept of thermodynamic annealing. We simulate the dynamics of various QCA logic-gate systems operated by this annealing method, and show that data processing in QCA systems can be carried out accurately by means of this annealing method. The applicability of QCA systems to non-Neumann parallel-processing computation is also described. (C) 1997 American Institute of Physics. [S0021-8979(97)01722-2]
\end{abstract}

\section{INTRODUCTION}

The quantum cellular automaton $(\mathrm{QCA})^{1-3}$ is a computation device that utilizes the energy-minimizing property of quantum cell systems. It was proposed by Lent and colleagues ${ }^{1}$ and has been receiving increasing attention because it affords the possibility of producing quantum largescale integrated circuits (LSIs) with large integration capability and low power consumption. To develop the QCA for practical use, we must find a way of operation that can make the QCA system compute successfully, or settle down exactly to its minimum-energy state. This article proposes such a method of operation, namely, an annealing method for operating QCA systems.

The concept of the QCA is as follows. To perform binary logic computation, first a many-body electron system is prepared that consists of a large number of simple identical quantum-dot elements (cells), where each cell has bistable states and changes its state through Coulomb interaction with its neighboring cells. In computing, some of the cells are used as input cells and others as output ones. The input binary data are provided for the system by fixing the states of the input cells. Under a given input, each cell adjusts its own state to those of all the others. After some interaction time, all the cells finally reach maximal consensus about their individual states, and the whole system then stabilizes in the minimum-energy state. By an ingenious arrangement or layout of the cells, the system can be so constructed that, when its minimum-energy state is achieved in response to a given input, the states of the output cells will represent the result of the computation. Thus the result of the computation can be retrieved by sensing the states of the output cells.

To develop QCAs into practical devices, we have to deal with the following two fundamental problems. The first is: can such a quantum-cell layout really exist, of which the minimum-energy state represents the result of the computa-

${ }^{\text {a)} E l e c t r o n i c ~ m a i l: ~ a k a z a w a @ s a p i e n s . h u e e . h o k u d a i . a c . j p ~}$ tion? Study of this problem by Lent and colleagues has shown that such a cell layout certainly can exist and that several elemental logic-gate circuits can probably be constructed by using QCAs. ${ }^{1-3}$

The second problem we must deal with is the subject of this article. It is: starting with a given initial state, how can we make the system settle down to its minimum-energy state? The initial state (i.e., just after data input) is not the minimum-energy state for the given input and, for computation, the QCA has to change its state to settle down to the minimum-energy state corresponding to the input. Because a many-body electron system in general has many states of locally minimum energy, we cannot be certain that the QCA system can achieve the state of globally minimum energy without being stuck in the local minima. The way of dealing with this problem depends on whether the electron system in the QCA is an adiabatic system or an open system. (The term "adiabatic system" means that the electrons in a QCA interchange no energy with the crystal lattice that forms the QCA device, and the "open system" means that the electrons do interchange energy with the crystal lattice.) Under adiabatic conditions, the electron system excited in energy by an input signal will maintain its "hot" properties during the computing operation. Consequently, the electron system will change its configuration freely without being stuck in the local minima, and successful QCA operation will result. This has been confirmed by Tougaw and Lent. ${ }^{3}$ They simulated signal transmission in a QCA under adiabatic conditions and showed that a signal can travel properly through the QCA in the form of an energy-excited part (called a "kink") in the electron system. Thus we can expect that QCAs will operate correctly under adiabatic conditions.

The situation is quite different if the electron system in a QCA is an open system. Under open-system conditions, the electron system excited by an input signal will lose its energy through interaction with the crystal lattice and transfer to lower energy levels, and consequently will fall into the local minima. Therefore, as things stand, the QCA cannot be 


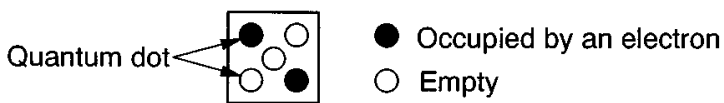

(a)

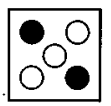

State 0

(b)
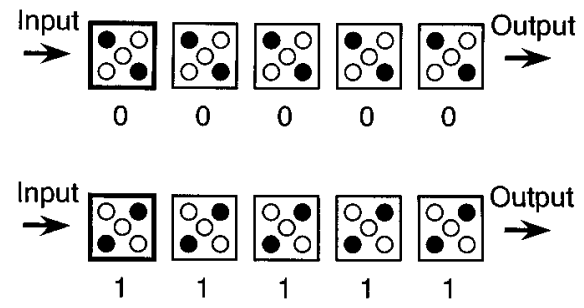

(c)

FIG. 1. Concept of the QCA circuit. (a) A unit cell consisting of five quantum dots with two electrons. (b) Two polarization states, 0 and 1. (c) Signal transmission through a cell array.

operated as desired. In most quantum-dot structures, the electron system interacts with the crystal lattice to interchange energy with lattice vibration, and so must be considered an open system. It is therefore essential to find a way of operation that can drive the system into the global-minimum energy state under open-system conditions.

In this article, we will propose an effective method for operating QCAs that can be applied to open-system conditions. In the following sections, first we calculate the potential energy of a QCA system as a function of the electron states. It will be shown that the QCA system has an energy function that has many local minima and therefore, under open-system conditions, cannot perform data processing as it stands (Sec. II). After that, to make the circuit operate correctly or converge to the minimum-energy state, we propose an annealing method for operating QCA systems. We will illustrate the concept of this method and then show by simulation that it can be used to operate QCAs (Sec. III). We will simulate data processing in various logic-gate circuits to show the effectiveness of the proposed method. The dependence of the processing speed on the size of the circuits will also be estimated (Sec. IV). Finally we will conclude by suggesting the applicability of QCAs to non-Neumann parallel-processing computation (Sec. V).

\section{ENERGY FUNCTIONS AND LOCAL MINIMA IN THE QCA}

\section{A. QCA structures}

The QCA is a computation system composed of many identical quantum cells. For details, see Refs. 1-3. Each cell consists of five dots located at the corners and the center of a square [Fig. 1(a)] and has two electrons that can tunnel be- tween any neighboring dots within the cell. (A fixed positive charge is also assumed to exist on each dot, to maintain charge neutrality in the cell.) Because of Coulomb repulsion, the two electrons tend to occupy diagonally opposed sites in the cell. As this gives the cell two states for polarization [Fig. 1(b)], we can encode a binary signal by manipulating the polarization. In an array of identical cells [Fig. 1(c)], the polarized state of an input cell (the left-end bold-edged cell in the figure) will induce the same polarization in all the cells in the array. From this it can be expected that a binary signal will be transferred through the array. By combining identical cells in appropriate layouts, various logic circuits can be constructed.

The energy of a QCA system depends on its electron arrangement (that is, which dots in the circuit are occupied by electrons). Lent and colleagues have calculated the value of the energy by solving the Schrödinger equation for various cell layouts to find the minimum-energy states. Based on the results of the calculation, they designed appropriate cell layouts for elemental logic-gate circuits. ${ }^{2,3}$ For the following discussions, we here give instances of their results [Figs. 2(a) through 2(d)]. Each gate circuit has one or more input cells (bold edged in the figures) and an output cell (double edged). Its cell layout is such that its energy will become the minimum for a specific electron arrangement in which the binary data of the input cells and of the output one satisfy the logic function to be implemented. Figures 2(a) and 2(b) illustrate a linear signal-transmission array and an angled one, for inputs " 0 "' and " 1 " with the corresponding minimum-energy electron arrangements. (In the figure, each electron is represented as a single particle. Strictly speaking, this is inaccurate because the electron wave function can extend over five dots within a cell. But in practice the electron wave functions for global and local minimum-energy states for a given input are localized mostly on two diagonally opposed dots in the cell, so we can represent the electron arrangement as shown in the figure.) Figure 2(c) shows an AND/OR gate circuit. It can switch between AND and OR operations by means of a control input: i.e., an AND operation when the control cell is fixed to 0 , or an OR operation when fixed to 1 . In this figure the electron arrangement for an AND operation with inputs $A=1$ and $B=0$ is shown. Figure 2(d) illustrates an inverter, with the electron arrangement for an input 0 .

There are three parameters that determine the circuit characteristics. They are: the nearest-neighbor distance between two corner dots in the same cell (denoted by $a$ in the figures), the nearest-neighbor distance between two dots in neighboring cells (denoted by $d$ ), and the fixed positive charge on each dot.

\section{B. Calculating the energy of a QCA system}

To analyze the behavior of a QCA system, we must first decide on a way to calculate the energy of the QCA system as a function of the electron arrangement.

The usual method for calculating QCA energy is the intercellular Hartree approximation. ${ }^{3}$ In this method, the state of each cell is determined by solving the Schrödinger equation for the cell, using a Hubbard-type Hamiltonian with a Coulomb interaction term and assuming the Hartree ap- 

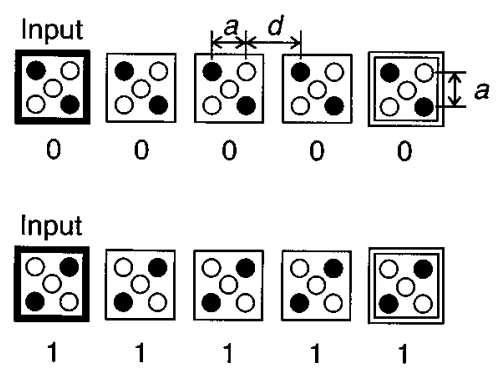

(a)
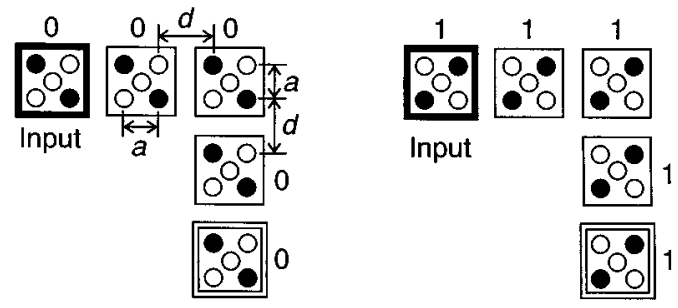

(b)

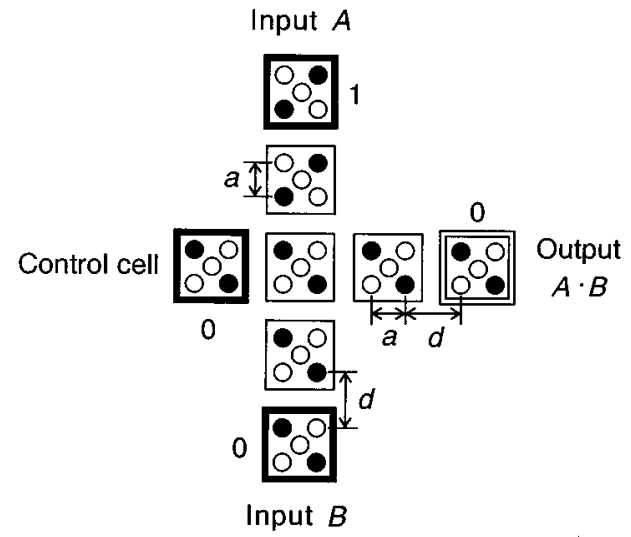

(c)

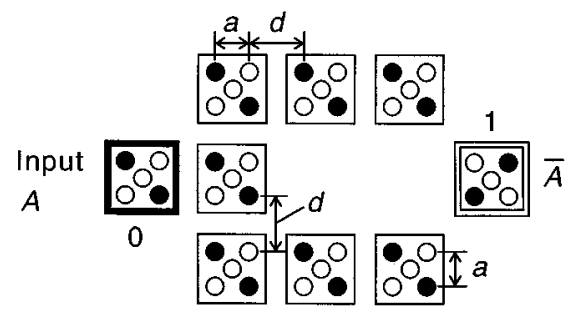

(d)

FIG. 2. Elemental logic-gate QCA circuits. Each has one or more input cells (bold edged) and an output cell (double edged). (a) Linear signaltransmission array. (b) Angled signal-transmission array. (c) AND/OR gate. (d) Inverter. Also illustrated is the minimum-energy electron arrangement for a given input.

proximation, which treats the charge in differing cells as the generator of a Hartree mean field. The minimum-energy state of the circuit as a whole is obtained by repeating self-

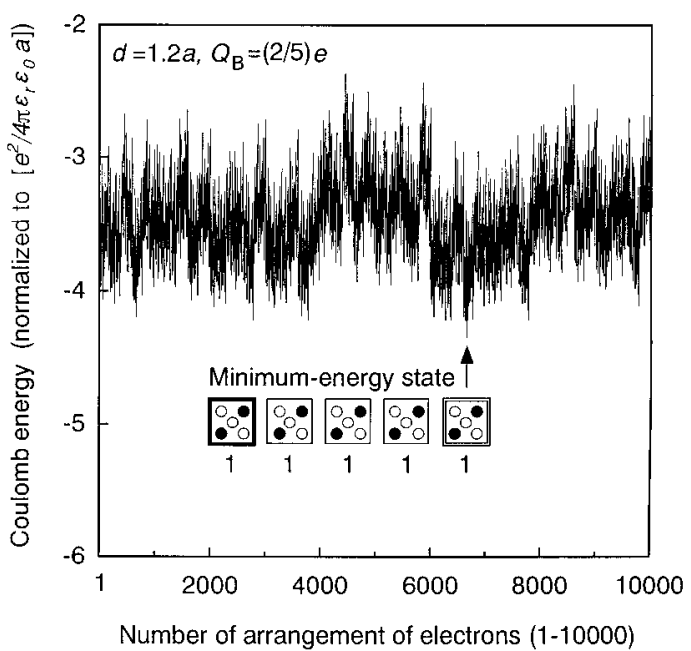

FIG. 3. Coulomb energy vs electron arrangement for a five-cell linear array circuit.

consistent calculation until the results of the calculation converge.

This method, however, cannot be used in our analysis. The present purpose is to study the process of QCA systems converging to a minimum-energy state. Hence, we have to consider the dynamics or time-dependent behavior of QCA systems under open-system conditions. As will be shown later, we have to perform many iterations to simulate the state transitions in a QCA system. An enormous and impractical amount of computing time is required to accomplish such dynamics simulation by means of Hartree-type approximation.

In the present analysis, we assume a simplified model for QCA circuits. In this model a QCA system can be regarded as a classical electrostatic system consisting of electrons and background positive charges, and its energy can be given simply as Coulomb potential energy. We also assume that the electron charge is a point charge, and that the size of each dot in cells is zero and therefore the background positive charge is a point charge. This simplification means that we ignore the effect of exchange coupling between electrons in a cell. This is not a problem, however, because the QCA system is generally designed for achieving distinct polarization of each cell, such that the exchange effect is small enough compared to the effect of Coulomb energy. ${ }^{2}$ (No exchange coupling exists between electrons in differing cells because the electrons cannot tunnel between differing cells.) Using this simplified model, we calculated in advance the electron arrangement that gives the minimum energy for each gate circuit in Figs. 2(a) through 2(d). The results agreed with those in Fig. 2 predicted by the intercellular Hartree approximation. This shows that the simplified model suffices for grasping the general situation of QCA systems.

\section{Local minima in QCA energy}

The energy of a QCA system is a function of the electron arrangement. For each of the logic-gate circuits given in Fig. 2, we have calculated the Coulomb potential energy for 
(a)

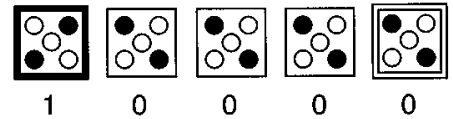

(b)
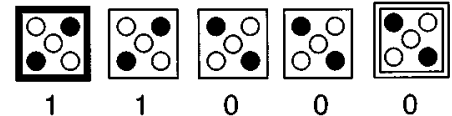

(c)
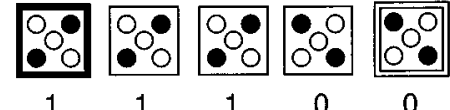

(d)
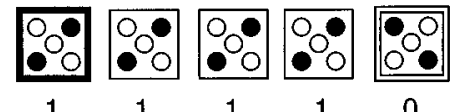

$E=-4.2182$

(e)

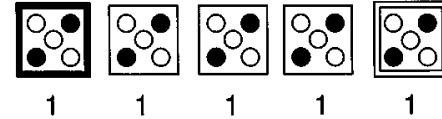

FIG. 4. A sequence of state transition for dominolike signal transfer through a five-cell linear array circuit, under open-system conditions. The corresponding normalized Coulomb energy $E$ is shown for each state.

all possible arrangements of electrons. We found that all the circuits have many local minima in their energy functions.

As an example, we illustrate in Fig. 3 the calculated energy function of a five-cell linear array circuit. We fixed the polarization of the input cell to state 1 , and calculated the energy for each possible arrangement of electrons in the other four cells. The horizontal axis of the figure indicates the number of the electron arrangement; one number corresponds to one arrangement of electrons; $10^{4}$ arrangements are possible because ten possible arrangements exist for two electrons in each of the four cells. In calculation, we assumed, following Ref. 2, that $d=1.2 a$ (for $a$ and $d$, see Sec. II A) and the background positive charge $Q_{B}$ on each dot was $(2 / 5) e$ ( $e$ is the elementary charge). In the figure, the energy is normalized to $e^{2} /\left(4 \pi \epsilon_{0} \epsilon_{r} a\right)\left(\epsilon_{0}\right.$ is the permittivity in vacuum, and $\epsilon_{r}$ a relative dielectric constant of the QCA circuit).

As illustrated in the figure, the energy of the circuit becomes minimum for a specific arrangement of electrons in which every cell takes the same polarization as that of the input cell- the desirable electron arrangement that transmits the input signal correctly. But it can also be seen that many local minima exist that have energy values close to that of the minimum-energy state. It is therefore not possible to be certain that, under open-system conditions, the circuit can always achieve the minimum-energy state, starting with a given initial state. In most cases the circuit will get stuck in a local minimum and therefore will not be able to transmit input signals.

This situation is illustrated simply in Fig. 4. Figures 4(a) through 4(e) depict the several states of the linear array circuit with the corresponding normalized energy values. Figure 4(a) corresponds to the initial state in which the input cell is fixed to state 1 and the other four cells are in state 0 . It might be expected that the polarized state of the input cell will induce the same polarization in all the cells in the array

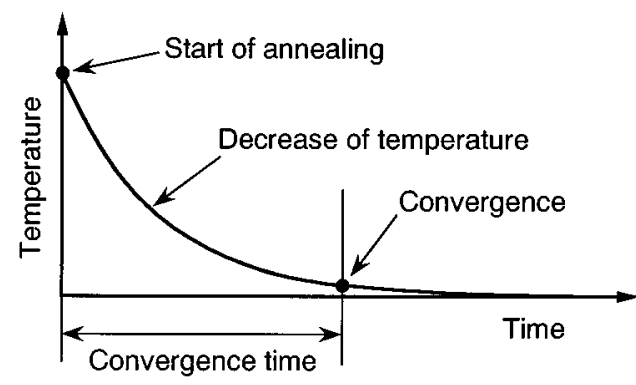

FIG. 5. Concept of the annealing procedure.

circuit, as illustrated in Figs. 4(b) through 4(e). But this cannot happen under open-system conditions. Because state $b$ has a higher energy than that of the initial state $a$, the circuit cannot make the transition to state $b$. And if it could, the circuit might not transfer from state $b$ to state $c$ because the energy is the same for both states.

\section{ANNEALING METHOD FOR OPERATING QCA CIRCUITS}

\section{A. Concept of QCA annealing}

Our objective in operating a QCA circuit is to reach a minimum-energy state without getting stuck in local minima. One effective way to avoid (or escape) local minima is to accept in a limited way energy-up transitions corresponding to an increase in the value of the energy. Tougaw and Lent reported that, in an adiabatic system, such excitation can be accomplished by input change (because the configuration changes from ground state to excited state), and the excitation energy is not dissipated until the end of dominolike signal transfer. ${ }^{3}$ Such transition cannot be guaranteed in an open system, where a QCA circuit is put in a heat bath and can interchange energy with it. Here, another way is needed.

We propose here an effective way of operating QCA circuits of open systems. It is the annealing operation method. The method consists of the following four steps (Fig. 5).

(1) Provide an input signal to a QCA circuit by fixing the polarization state of the input cells.

(2) Increase the temperature of the heat bath to a maximum value at which the QCA circuit changes its electron arrangement randomly.

(3) Carefully decrease the temperature of the heat bath until the QCA circuit arranges its electrons in the minimumenergy arrangement (or until the QCA circuit reaches convergence).

(4) Read the result of the computation by detecting the polarization state of the output cell.

If the lowering of the temperature is done slowly enough, the QCA circuit can reach thermal equilibrium at each temperature, and therefore can approach a minimumenergy state with decrease in temperature. (Here the "temperature" need not be thermal temperature; any factor that can activate a QCA circuit, e.g., light and electromagnetic waves, can be used.) 
Originally, the term "annealing'" was confined mostly to the metallurgical process for obtaining a perfect metal crystal without deformations and dislocations-heating a body of metal to near its melting point and then cooling it slowly to room temperature. In this study, we adopted annealing as an effective method for achieving successful QCA operation. In the following sections, we will show by computer simulation that a QCA circuit can carry out correct data processing through settling down, or converging successfully, to its minimum-energy state by means of annealing.

\section{B. Simulated annealing}

We simulated the process of QCA annealing by means of Monte Carlo techniques. In advance of the annealing, the cooling schedule, i.e., a decrement function for lowering the temperature, must be determined. Among several cooling schedules, ${ }^{4-7}$ we used the natural cooling given by $T$ $=T_{0} \exp (-\rho t)$, where $T$ is the temperature, $T_{0}$ is an initial value of the temperature, $\rho$ is a cooling-speed coefficient, and $t$ is time. The values of $T_{0}$ and $\rho$ govern the convergence of a QCA circuit during annealing. To achieve the convergence successfully within the shortest annealing time, the optimum set of $T_{0}$ and $\rho$ has to be determined. This depends on the structure of the QCA circuit to be operated, and can be determined only through simulation.

The algorithm for the simulation is as follows.

\section{Initial setup for the annealing}

Given a QCA circuit to be operated, provide input data to the circuit by fixing the polarization of input cells throughout the annealing procedure (the other cells are unrestrained). Choose appropriate values for the cooling-schedule parameters $T_{0}$ and $\rho$. Choose the starting state of the QCA circuit, and set $t=0$. (In the following, a state means an arrangement of electrons in the QCA circuit.)

\section{Monte Carlo iteration of the annealing process}

(a) Step 1. Compute the energy $E_{0}$ for the current state of the QCA circuit. Then enumerate all possible subsequent states and compute the energy $E_{i}$ for each subsequent state $i$. (A subsequent state means a state into which the current state can be transformed by one tunneling of an electron. The co-tunneling phenomenon is ignored for simplicity.)

(b) Step 2. Compute the energy difference $\Delta E_{i}\left(=E_{i}\right.$ $-E_{0}$ ) for each subsequent state. From the value of $\Delta E_{i}$, calculate the waiting time for a tunneling process corresponding to each subsequent state. The waiting time $\tau\left(\Delta E_{i}\right)$ is given as follows (see Appendix):

$$
\tau\left(\Delta E_{i}\right)=\left\{\begin{array}{l}
\tau_{0} \ln (1 / r) \quad\left(\text { if } \Delta E_{i} \leqslant 0\right) \\
\tau_{0} \exp \left(\Delta E_{i} / k_{B} T\right) \ln (1 / r) \quad\left(\text { if } \Delta E_{i}>0\right),
\end{array}\right.
$$

where the constant $\tau_{0}$ is the mean waiting time for tunneling, $r$ is a uniform random number $(0<r<1)$ generated for each tunneling event, $k_{B}$ is the Boltzmann constant, and $T$ is the temperature given by the cooling schedule $T=T_{0}$ $\exp (-\rho t)$.

If $\Delta E_{i}>0$, the waiting time $\tau_{0} \ln (1 / r)$ is multiplied by a factor of $\exp \left(\Delta E_{i} / k_{B} T\right)$. This corresponds to the fact that a tunneling process for $\Delta E_{i}>0$ needs the assistance of thermal energy or of a phonon. (The factor $\exp \left(\Delta E_{i} / k_{B} T\right)$ corresponds to the reciprocal of acceptance probability $\exp \left(-\Delta E_{i} / k_{B} T\right)$ in the simulated-annealing process proposed by Metropolis and colleagues ${ }^{8}$ to simulate the evolution of a solid in a heat bath to thermal equilibrium.)

The mean waiting time $\tau_{0}$ depends on the distance tunneled by an electron. Two kinds of tunneling can occur within a QCA cell: the tunneling between a corner dot and the center dot (short-distance tunneling) and the tunneling between two corner dots (long-distance tunneling). We named the mean waiting time for the former the Monte Carlo time unit. The latter tunneling is expected to be less frequent than the former, so we assumed the mean waiting time for the latter to be ten times that of the former.

(c) Step 3. After calculating the waiting time $\tau\left(\Delta E_{i}\right)$ for all possible tunnelings, take the tunnel event that has the shortest waiting time, and accept the corresponding subsequent state as the current state. Then put the time forward by $\tau\left(\Delta E_{i}\right)$ and return to step 1 to repeat the iterations.

\section{Judgment of the convergence}

Steps 1 through 3 of the Monte Carlo iteration above are repeated until it appears that a convergence has been achieved. We need suitable criteria for deciding to stop the procedure. In the present calculation, we judge that the states of the QCAs have converged if the current state has not been updated for 50000 Monte Carlo time units. We then check to see whether the converged state is the minimum-energy state. If it is, the annealing has succeeded; if not, it has failed. We call the above annealing procedure a trial.

For a given QCA circuit, we repeated 100 trials, using a different series of random numbers for $r$, and then judged the annealing to be successful if all the trials were successful.

\section{Determination of the optimum values of $T_{0}$ and $\rho$}

We must find the optimum set of cooling-schedule parameters (the initial temperature $T_{0}$ and the cooling-speed coefficient $\rho$ ) that achieves the successful convergence in the shortest annealing time. The procedure for finding the optimum $T_{0}-\rho$ set is as follows. We first select a large value of $T_{0}$ and repeat the simulated annealing for various values of $r$ to determine the $\rho$ value that gives the shortest annealing time for the given $T_{0}$ value. Then we reduce the $T_{0}$ value by a slight decrement and repeat the same calculations. By repeating this procedure, we can find the optimum $T_{0}-\rho$ set for that particular QCA circuit.

Many iterations or much computing time is required to determine the optimum $T_{0}-\rho$ set for a given circuit. Therefore we determined the optimum set only for the AND/OR gate circuit [Fig. 2(c), the most complex circuit among the elemental QCA circuits shown], and used that value of $T_{0}$, $6.69 \mathrm{~K}$, for all other circuits (the $\rho$ value was optimized for each circuit).

\section{The result for a linear array circuit}

We first simulated the annealing process for linear signal-transmission array circuits. The result of a trial for a 


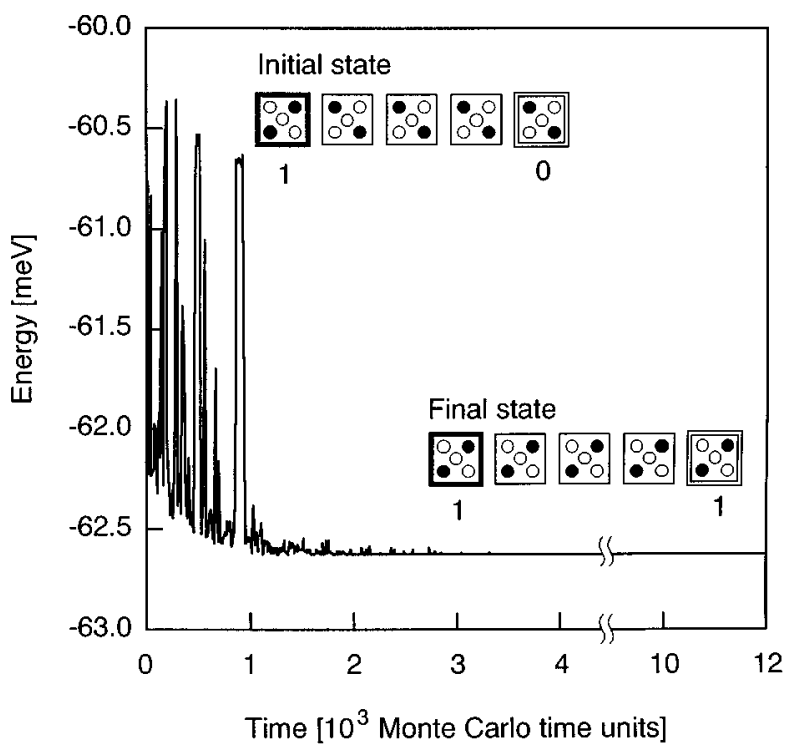

FIG. 6. Simulated annealing for a five-cell linear array circuit. Initial state: the input cell is 1 and the other cells are 0. Final state: all the cells are 1.

five-cell linear array is shown in Fig. 6. The energy transition resulting in convergence is illustrated as a function of time normalized to the Monte Carlo time unit. The polarization state of the input cell (the left-end bold-edged cell) was fixed to 1 throughout the annealing procedure, and at the start $($ time $=0)$ the other four cells were set to 0 . The scheduling parameters were $T_{0}=6.69 \mathrm{~K}$ and $\rho=5 \times 10^{-4}$ (1/Monte Carlo time unit). The structure parameters were assumed to be $a=10 \mathrm{~nm}, d=12 \mathrm{~nm}$, and $\epsilon_{r}=10$.

We calculated the energy of the circuit for every transient state, but for simplicity plotted in the figure the mean values for every 10 Monte Carlo time units. As more than 2,500 tunneling events or state transitions occurred, it was not possible to plot each event.

It can be seen in the figure that after many state transitions the electron arrangement in the QCA circuit finally converges to the energy-minimum state. Consequently, the QCA circuit was able to transmit the input signal correctly. The mean convergence time for 100 annealing trials was 10100 Monte Carlo time units (apart from the 50000 Monte Carlo time units expended in judging the convergence).

In this QCA circuit, the energy difference between the minimum-energy state and the second-lowest energy state, under the given input, is $1.1 \mathrm{meV}$, corresponding to $13 \mathrm{~K}$. Therefore, to prevent thermal agitation, the temperature at the final stage of the annealing has to be much lower than 13 $\mathrm{K}$. The maximum value of final temperature, which varied from trial to trial, for 100 trials, was $1.03 \mathrm{~K}$.

It is possible to convert the Monte Carlo time unit into physical time, provided that the value of the mean waiting time for tunneling between a corner dot and the center dot in a cell can be known. Experiments for several quantum structures have shown that the tunneling interaction time between two coupled dots can be $0.05-2 \mathrm{ps},{ }^{9-11}$ so the unit of Monte Carlo time in QCA circuits can be expected to be similar in value. Assuming the unit to be $1 \mathrm{ps}$, the mean convergence time can be estimated at $10.1 \mathrm{~ns}$.

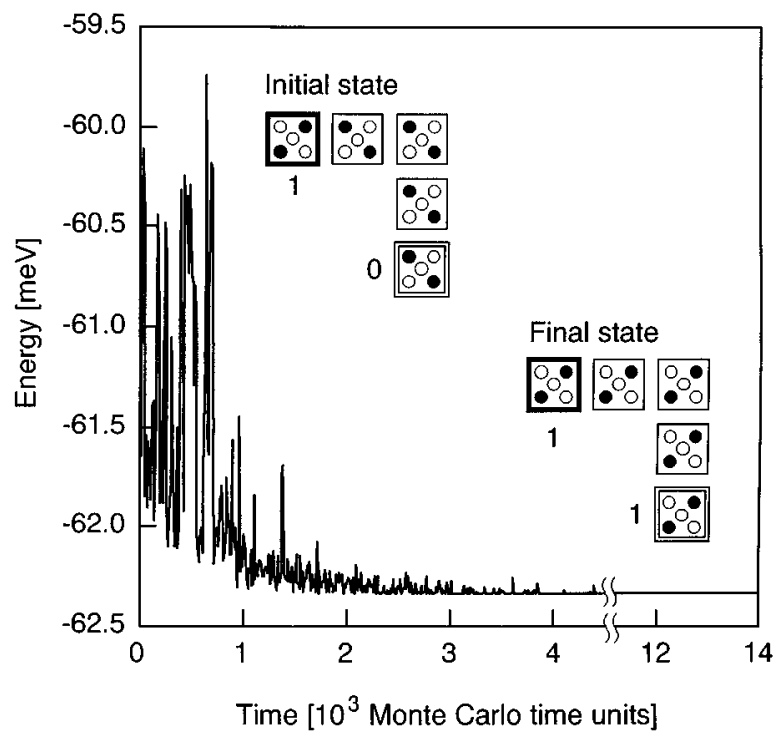

FIG. 7. Simulated annealing for a five-cell angled array circuit. Initial state: the input cell is 1 and the other cells are 0. Final state: all the cells are 1.

\section{SIMULATED ANNEALING FOR ELEMENTAL LOGIC-GATE CIRCUITS}

This section illustrates the results of simulated annealing for elemental logic-gate circuits (an angled signaltransmission array, an inverter, and an AND/OR gate) to show the effectiveness of annealing in QCA operation. In the simulations, the polarization of an input cell (or cells) was fixed to a given state ( 1 or 0$)$ throughout the annealing procedure. At the start (time $=0$ ), the electrons in the other cells were set into the arrangement that would give the minimum energy if the input cell were in an opposite state. (The structure parameters were $a=10 \mathrm{~nm}, d=12 \mathrm{~nm}$, and $\epsilon_{r}=10$ for all sample circuits. A background positive charge $Q_{B}$ $=(2 / 5) e$ on each dot was assumed. $)$

\section{A. Angled array circuit}

The result of a trial of the annealing for a five-cell angled array is illustrated in Fig. 7. Plotted are the mean energy values for every ten Monte Carlo time units. The annealing parameters were $T_{0}=6.69 \mathrm{~K}$ and $\rho=3 \times 10^{-4}$ (1/Monte Carlo time unit). After many state transitions, the circuit converged to the minimum-energy state, and thereby transmitted the input signal correctly. The average convergence time for 100 trials was 12500 Monte Carlo time units (12.5 ns for a unit of $1 \mathrm{ps}$ ).

\section{B. Inverter circuit}

The annealing process for an inverter circuit (a NOT gate) is illustrated in Fig. 8. Again, successful convergence was obtained, and consequently the circuit was able to perform the NOT operation correctly. The annealing parameters were $T_{0}=6.69 \mathrm{~K}$ and $\rho=5 \times 10^{-5}$ (1/Monte Carlo time unit). The mean convergence time for 100 trials was 56500 Monte Carlo time units (56.5 ns for a unit of $1 \mathrm{ps}$ ). 


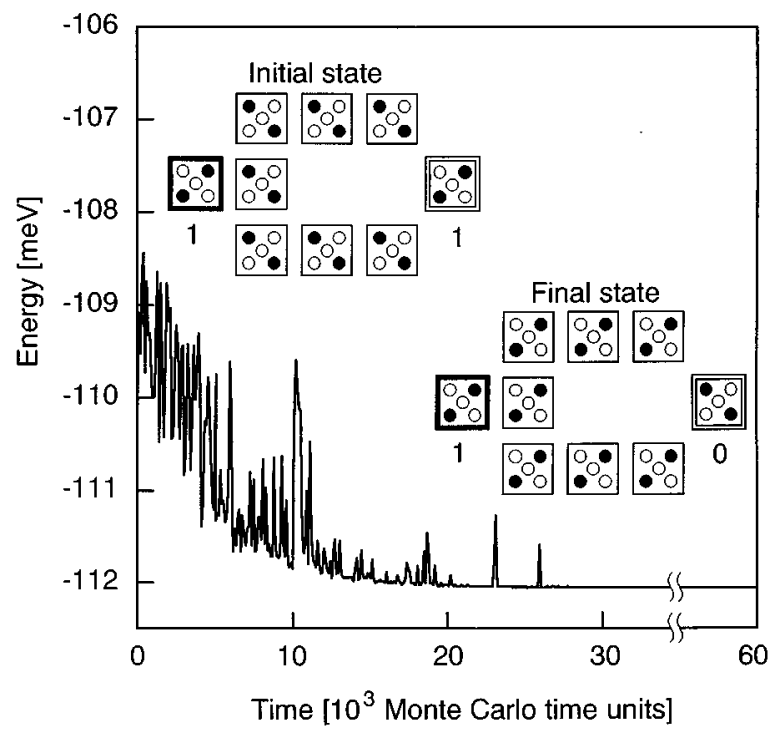

FIG. 8. Simulated annealing for an inverter circuit. The input cell is fixed to 1. Initial state: the output is 1 . Final state: the output is 0 .

\section{AND/OR gate circuit}

As described in Sec. II A, the AND/OR gate circuit operates as an AND gate if the control cell is fixed to 0 and as an OR gate if the control cell is fixed to 1 . We have simulated and confirmed the logic operation for both AND and OR operations for all possible input combinations. We here illustrate in Fig. 9 the result for AND operation with one input set at 1 and the other input set at 0 . The annealing parameters were $T_{0}=6.69 \mathrm{~K}$ and $\rho=6.7 \times 10^{-6}$ (1/Monte Carlo time unit). Through annealing, the circuit converged to the minimum-energy state at which the polarization of the output cell was 0 , and thereby performed the AND logic

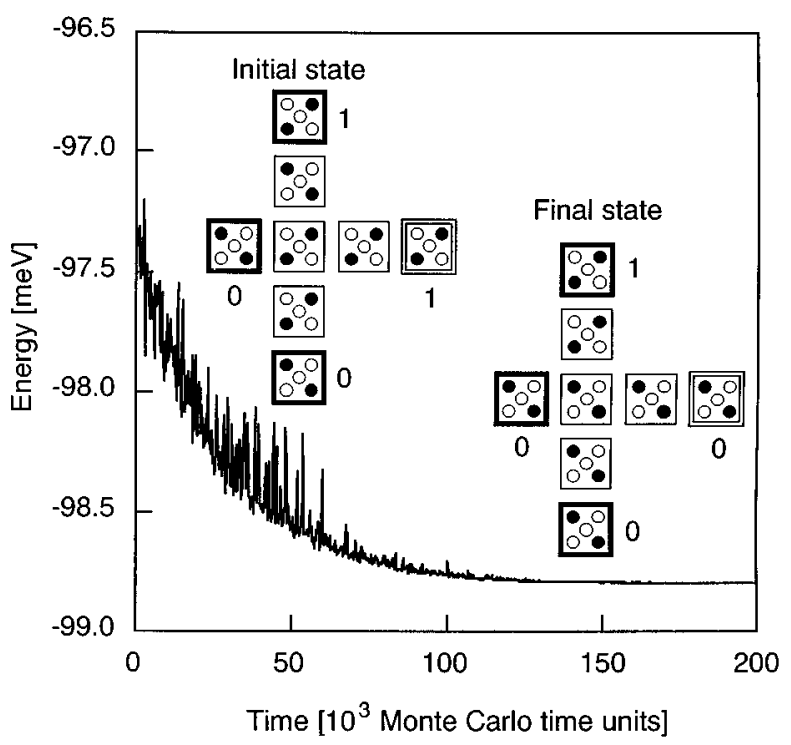

FIG. 9. Simulated annealing for an AND circuit. The control cell is fixed to 0 , and two inputs are fixed to 1 and 0 . Initial state: the output is 1 . Final state: the output is 0 .

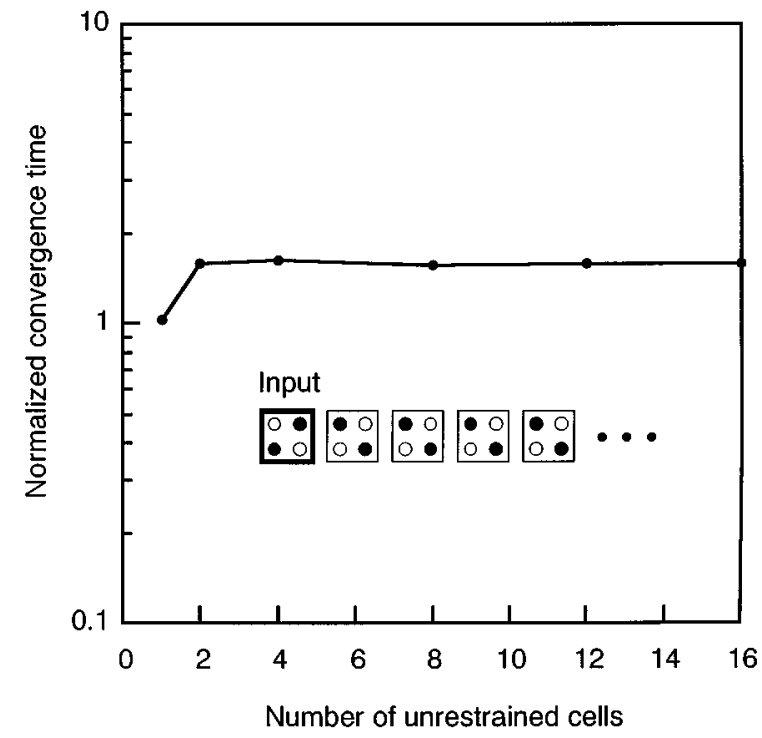

FIG. 10. Convergence time vs number of unrestrained cells for a linear array circuit. The convergence time result is normalized to that of the twocell circuit (one-unrestrained-cell circuit).

operation correctly. The mean convergence time for 100 trials was 186000 Monte Carlo time units (186 ns for a unit of $1 \mathrm{ps})$.

\section{Relation between convergence time and circuit size}

In previous sections, we confirmed that data processing or logic operation in QCA can be carried out successfully by use of the annealing method. But if the convergence time increases rapidly with increase in the number of cells, it will be impossible to operate large-sized QCA circuits within a practical span of time. An increase in the number of cells leads to an exponential increase in the number of possible electron arrangements, so the convergence time may also increase exponentially with the number of cells.

To investigate this problem, we carried out simulated annealing for a linear array circuit, changing the number of cells from 2 to 17. (In this calculation, we assumed four dots-one at each corner-in a cell, instead of a five-dot cell. This is because simulation for a large-sized five-dot-cell array requires enormous computing time.) The result is illustrated in Fig. 10, which shows the convergence time as a function of the number of unrestrained cells. Contrary to our expectations, the convergence time showed hardly any dependence on the number of unrestrained cells. Though a definitive conclusion cannot be drawn before other QCA circuits have been investigated, it seems that the convergence time for the annealing does not increase rapidly with an increase in the size of the QCA circuit.

\section{APPLICATION TO NON-NEUMANN COMPUTATION}

The proposed annealing method for QCAs will open up novel application fields of QCAs, in addition to digital logic computation. An example is non-Neumann parallel processing computation for solving combinatorial problems. For example, consider the following problem: given a well-formed 
Boolean expression, is there a truth assignment for the set of variables that satisfies the expression? (For instance, given the Boolean expression $(X 1+X 2+X 3)(X 1+\overline{X 2}+X 3)(\overline{X 1}$ $+\overline{X 2}+\overline{X 3}$ ), is there a combination of the values of variables $X 1$ through $X 3$ that makes the logical value of the expression be 1?) This problem, the satisfiability problem, belongs to the class of NP complete (nondeterministic polynomialtime complete), and is intractable for ordinary digital computers because the computational steps for problem solving increase exponentially with the increase in the size of the problem. But it can be expected that the QCA will provide an ingenious device that can quickly solve this kind of problem. We first prepare a QCA circuit that corresponds to the given Boolean expression, then fix the output cell to a state 1 and leave the input cells unrestrained (the states of the input cells correspond to the binary values of variables). After that we anneal the QCA circuit into an equilibrium state with global-minimum energy, then check to see whether or not the set of the final states of the input cells is a satisfying truth assignment for the given Boolean expression. If it is, the Boolean expression is satisfiable and the input cell states indicate a truth assignment for the set of variables. If it is not, the given expression is not satisfiable.

\section{CONCLUSION}

The QCA is a device that performs computation by utilizing the energy-minimizing property of quantum-cell structures. A key requirement in operating a QCA system successfully is to make the system converge exactly to its global-minimum energy state without getting stuck in local minima. For this purpose, we proposed an annealing method for operating QCA systems under open-system conditions and performed simulated annealing to show the effectiveness of the proposed method. We confirmed that it is possible to successfully operate elemental QCA gate circuits such as the linear array, the angled array, the inverter, and AND/OR gates. We also estimated the dependence of the processing speed on the size of the circuits and found that it showed no decrease in speed with increased circuit size. This indicates the possibility of operating a large QCA within a practical time span. The proposed annealing method for QCAs will open up novel application fields for QCAs, in addition to digital logic computation. As an example, a non-Neumann parallel processing computation for solving combinatorial problems was described.

Finally, it should be also stressed that the proposed annealing method is also useful for other QCA-type systems that utilize many-body interaction effects, such as the spinpolarized antiferromagnetic devices and the multistable quantum-well matrix devices. ${ }^{12-15}$

\section{ACKNOWLEDGMENTS}

The authors are grateful to Dr. Hiroyuki Tamura (NTT Basic Research Laboratories) for discussions on the validity of the simplified model for QCA circuits. This work was supported by a Grant-in-Aid for Scientific Research on Priority Areas- "Single-Electron Devices" from the Ministry of Education, Science, Sports, and Culture.

\section{APPENDIX}

In the following, we consider the electron tunneling between two dots (one dot is occupied by an electron and the other empty) in the same cell and calculate the waiting time for electron tunneling from the occupied dot to the empty one.

The probability $p$ that tunneling will occur within a period of time $=t$ to $t+\Delta t$ is given by a product of the probability that no tunneling will occur from time $=0$ to $t$ and the probability that tunneling will occur within the time duration of $\Delta t$. It is expressed as

$$
\begin{aligned}
p & =\{1-\Gamma(\Delta E) \Delta t\}^{t / \Delta t} \Gamma(\Delta E) \Delta t \\
& =\left[\{1-\Gamma(\Delta E) \Delta t\}^{1 /(-\Gamma(\Delta E) \Delta t)}\right]^{-\Gamma(\Delta E) t} \Gamma(\Delta E) \Delta t,
\end{aligned}
$$

where $\Gamma(\Delta E)$ is the mean tunneling rate, which is a function of the QCA-circuit energy difference $\Delta E$ resulting from the tunneling. Assuming that $\Delta t$ is sufficiently short (and rewriting $\Delta t$ as $d t$ ), we have

$$
p=\exp \{-\Gamma(\Delta E) t\} \Gamma(\Delta E) d t .
$$

Then the probability $P_{t}$ of tunneling occurring within a period of time $=0$ to $t$ is given by

$$
\begin{aligned}
P_{t} & =\int_{0}^{t} \exp \{-\Gamma(\Delta E) t\} \Gamma(\Delta E) d t \\
& =1-\exp \{-\Gamma(\Delta E) t\}<1) .
\end{aligned}
$$

Determining whether or not tunneling will occur is done by using a random number. That is, a uniform random number $\xi$ $(0<\xi<1)$ is selected, and if $\xi \leqslant P_{t}$, then tunneling will occur. This condition is given as

$$
\xi \leqslant 1-\exp \{-\Gamma(\Delta E) t\} .
$$

The waiting time for tunneling $\tau(\Delta E)$ is the minimum value of time $t$ that satisfies the inequality above. Therefore we have

$$
\tau(\Delta E)=\Gamma(\Delta E)^{-1} \ln (1 / r),
$$

where $r=1-\xi$. We assume that the tunneling for $\Delta E>0$ needs the assistance of a phonon having Boltzmann distribution in energy space, while the tunneling for $\Delta E \leqslant 0$ needs no assistance. Then the tunneling rate is given as

$$
\Gamma(\Delta E) \propto\left\{\begin{array}{lll}
1 & (\Delta E \leqslant 0) \\
\exp \left(-\Delta E / k_{B} T\right) & (\Delta E>0)
\end{array} .\right.
$$

Therefore we have

$$
\tau(\Delta E)=\left\{\begin{array}{l}
\tau_{0} \ln (1 / r) \quad(\Delta E \leqslant 0) \\
\tau_{0} \exp \left(\Delta E / k_{B} T\right) \ln (1 / r) \quad(\Delta E>0),
\end{array}\right.
$$

where $\tau_{0}$ is the mean waiting time for tunneling (an inverse of the mean tunneling rate), which depends on the structure and the material of the QCA unit cell.

\footnotetext{
${ }^{1}$ C. S. Lent, P. D. Tougaw, and W. Porod, Appl. Phys. Lett. 62, 714 (1993).

${ }^{2}$ C. S. Lent and P. D. Tougaw, J. Appl. Phys. 74, 6227 (1993).

${ }^{3}$ P. D. Tougaw and C. S. Lent, J. Appl. Phys. 80, 4722 (1996).

${ }^{4}$ E. Aarts and J. Korst, Simulated Annealing and Boltzmann Machines (Wiley, Chichester, 1989).
} 
${ }^{5}$ S. Geman and D. Geman, IEEE Trans. Pattern. Anal. Mach. Intell. 6, 721 (1984).

${ }^{6}$ S. Kirkpatrick, C. D. Gelatt, Jr., and M. P. Vecchi, Science 220, 671 (1983).

${ }^{7}$ V. Çerny, J. Optim. Theory Appl. 45, 41 (1985).

${ }^{8}$ N. Metropolis, A. W. Rosenbluth, M. N. Rosenbluth, and A. H. Teller, J. Chem. Phys. 21, 1087 (1953).

${ }^{9}$ A. Nakano, R. K. Kalia, and P. Vashishta, Appl. Phys. Lett. 62, 3470 (1993).
${ }^{10}$ N. Tsukada, M. Gotoda, and M. Nunoshita, Phys. Rev. B 50, 5764 (1994).

${ }^{11}$ W. Potz and J. Zhang, Phys. Rev. B 45, 11496 (1992).

${ }^{12}$ K. Obermayer, W. G. Teich, and G. Mahler, Phys. Rev. B 37, 8096 (1988); 37, 8111 (1988).

${ }^{13}$ M. Biafore, Physica D 70, 415 (1994).

${ }^{14}$ K. Nomoto, R. Ugajin, T. Suzuki, and I. Hase, J. Appl. Phys. 79, 291 (1996).

${ }^{15}$ S. Bandyopadhyay and V. Roychowdhury, Jpn. J. Appl. Phys., Part 135 , 3350 (1996). 